\title{
Care of the Stroke Patient: Routine Management to Lifesaving Treatment Options
}

\author{
George A. Lopez • Arash Afshinnik • Owen Samuels
}

Published online: 12 July 2011

(C) The American Society for Experimental NeuroTherapeutics, Inc. 2011

\begin{abstract}
The management of the acute ischemic stroke patient spans the time course from the emergency evaluation and treatment period through to the eventual discharge planning phase of stroke care. In this article we evaluate the literature and describe what have become standard treatments in the care of the stroke patient. We will review the literature that supports the use of a dedicated stroke unit for routine stroke care which has demonstrated reduced rates of morbidity and mortality. Also reviewed is the use of glycemic control in the initial setting along with data supporting the use of prophylactic treatments options in order to aide in the prevention of life threatening medical complications. In addition, lifesaving treatments will be discussed in light of new literature demonstrating reduced mortality in large hemispheric stroke patients undergoing surgical decompressive surgery. Both medical and surgical treatment options are discussed and compared.
\end{abstract}

Keywords Stroke $\cdot$ Hyperglycemia $\cdot$ Hyperosmolar .

Hemicraniectomy Stroke unit

\section{Stroke Units}

After initial treatment decisions, usually occurring in the emergency rooms of most hospitals, patients with acute

Electronic supplementary material The online version of this article (doi:10.1007/s13311-011-0061-1) contains supplementary material, which is available to authorized users.

G. A. Lopez $(\bowtie)$

Department of Neurology, Houston Health Science Center, University of Texas,

Houston, TX 77030, USA

e-mail: george.a.lopez@uth.tmc.edu

A. Afshinnik · O. Samuels

Division of Neurosciences Critical Care, Emory Healthcare,

Emory University,

Atlanta, GA, USA ischemic stroke are admitted to the hospital to 1 of various different locations. Depending on the severity of the acute stroke, a patient may be admitted to a general intensive care unit (ICU) or a specialized neuroscience ICU. Once a patient is stabilized, usually 24 hours after tissue plasminogen activator has been administered, then the patient may be transferred out of ICU to a general medical room. On the other hand, if a hospital has a specialized stroke unit (SU) the patient may be admitted to the SU, initially bypassing the ICU or neuroscience ICU [1]. Stroke units tend to be physically located on distinct floors or units in the hospital with dedicated nursing staff, therapists, social workers, and case management teams. Organized inpatient care is not a new concept with literature dating back several decades [2,3].

It has been debated for several years whether dedicated and organized units such as SUs can provide meaningful improvements in tangible ways [3]. The question is whether patients treated in a SU fare better in terms of recovery, mortality, hospital costs, and eventual disposition than those not treated in a specialized unit. In a recent review of observational studies implementing organized stroke units, Seenan et al. [4] found that those patients who were managed in an SU showed reduced odds of death or poor outcome. Within all subgroups analyzed, there was a significant reduction in the risk of death associated with SU care. It can be argued, however, that these types of significant findings may only be applicable to SUs that are participating in clinical trials and may not be applicable in day-to-day routine clinical practice. The studies cited by Seenan et al. [4] in their analysis of SU care are more likely to reflect routine clinical care. The association found with the outcomes and use of SUs was comparable to the data obtained from the SUs that were involved in clinical trials.

A systematic review of all available trials $[5,6]$ by the Cochrane Review Database, which previously described 
the range of characteristics of SU care, addressed whether improving the organization of inpatient stroke care could bring about improvements in patient outcomes. Organized inpatient SU care, when compared with conventional care, was characterized by coordinated multidisciplinary rehabilitation, programs of education and training of strokes, and specialization of medical and nursing staff. SU care was associated with a long-term reduction of death and of the combined poor outcomes of death or dependency and death or institutionalization. The observed benefits were not restricted to any particular subgroup of patients or model of SU care. Another benefit shown was that there was no increase in length of stay. This is a powerful finding as a prior study evaluating cost and resource utilization in an organized stroke unit at a tertiary academic center found that the largest determinant of cost for a stroke patient was hospital length of stay [7]. Other determinants found were stroke severity, cardiac disease, male gender, and the use of heparin. Acute rehabilitation units may or may not be incorporated into the $\mathrm{SU}$, or they may be free-standing facilities distinct from the hospital. Incorporating acute and rehabilitation services into a combined SU has recently been shown to reduce length of stay and also improve outcomes in patients with acute stroke [8].

The findings of improved outcome measures and financial costs have mainly been limited to defined SUs and not necessarily to hospitals that use and maintain stroke pathways and order sets. In a recent retrospective study from a single institution examining patient outcomes, a clinical pathway for acute stroke patients had no benefit to the functional outcome of the patients [9]. This is in agreement with a systematic review of several studies both randomized and nonrandomized, showing that clinical pathways do not improve mortality or dependency for patients with stroke [10]. However, despite the lack of evidence base to support the use of pathways and order sets for stroke patients, clinical pathways are still recommended as a means of promoting best clinical practice in hospital care [11].

In summary, there is clear evidence from randomized trials and from systematic reviews of published trials for the establishments of dedicated stroke units to improve patient outcomes and increase survival rates. There are also financial benefits for the hospital and healthcare systems to implement such units for acute stroke patients. In addition, these findings of the benefits of SUs may also be applicable to stroke patients with a primary intracerebral hemorrhage [12]. In a randomized trial with patients presenting within $24 \mathrm{~h}$ of their hemorrhage, the authors found a reduced mortality rate at 30 days after admission to a dedicated $\mathrm{SU}$ at a single center.

\section{Prophylaxis for Venous Thromboembolism}

Stroke is associated with a high risk for venous thromboembolism (VTE). There are several reasons why stroke patients are predisposed to developing deep venous thrombosis and subsequent pulmonary embolism (PE). Factors such as age $>70$ years, and having a low Barthel Index severity score along with the presence of a hemiparesis with immobility all place the acute stroke patient at risk [13].

The true incidence of VTE is unknown, with published rates ranging anywhere from 5 to $80 \%$ of stroke patients not treated with thromboprophylaxis [13]. The rate of discovering a deep venous thrombosis (DVT) is also directly dependent on the screening test used and on whether the test was obtained based on clinical suspicion or not. For example, using $\mathrm{I}^{125}$ fibrinogen scanning, the rate of DVT ranges from 30 to $80 \%$, to as low as $5 \%$ using Doppler ultrasound $[14,15]$. Even in patients who were treated with thomboprophylaxis, the incidence of VTE can be significant. In a recent study, Kelly et al. [16] used thrombus imaging by magnetic resonance imaging. They prospectively assessed for the incidence of DVT and PE and found $18 \%$ of patients had a proximal DVT and as great as $12 \%$ had already had a PE [16]. With such a wide range of reported incidence, direct comparison between studies is problematic.

The efficacy of prophylaxis in preventing VTE in medical patients has been established [17], but it is less established in patients with stroke, because of differing study design [13] and the short duration of prophylaxis used. Most studies used treatment duration of only 2 weeks; however, the risk of PE can remain for $>4$ weeks after a stroke. Various pharmacological and nonpharmacological means have been studied in acute stroke patients, including aspirin, unfractionated heparin, the heparinoids, and low molecular weight heparin, all with varying degrees of effectiveness.

The efficacy of aspirin in preventing VTE in stroke patients has been studied in a few large trials. In the Chinese Acute Stroke Trial, treatment with aspirin did not reduce the incidence of PE [18]. In another large trial of stroke patients, the International Stroke Trial (IST) [19], the incidence of PE was similar in those treated with aspirin $(0.7 \%)$ vs those without aspirin $(0.9 \%)$ [19]. A Cochrane Review found a relative risk reduction of PE of $29 \%$ with the use of antiplatelets [20]. Aspirin use alone for the prevention of VTE, however, is not recommended [17].

Trials of subcutaneous unfractionated heparin for VTE prophylaxis in stroke patients have also had varied conclusions. In the largest randomized trial for VTE prophylaxis, the use of unfractionated heparin was not associated with a reduction of $\mathrm{PE}$ in 2 different dosing 
regimens [19]. The incidence of PE was only reduced if aspirin $(300 \mathrm{mg})$ was also used with subcutaneous heparin. However, this came with an associated 4-fold increased rate of intracranial hemorrhage $(1.2 \%$ vs $0.3 \%)$. In a much smaller randomized trial, using subcutaneous unfractionated heparin 3 times per day, there was a reduction in both the rates of DVT $(22 \%$ vs $73 \%)$ and PE postmortem $(10 \%$ vs 46\%) [21]. However, in a more recent trial using the same dosing regimen, there was no difference in the incidence of either DVT or PE between groups and no difference in the rate of intracranial hemorrhage [15].

In addition to the studies examining unfractionated subcutaneous heparin, there have been several studies that have compared different types and different doses of low molecular weight heparin (LMWH). In the largest of these trials, the Trial of Org 10172 in Acute Stroke Treatment (TOAST) [22] danaparoid was shown to decrease the incidence of DVT $(0.3 \%$ vs $1.6 \%)$, but it did not decrease the rate of PE [22]. There have been several other smaller studies comparing LMWH to either a placebo or to unfractionated heparin, which showed a decreased rate of DVT or PE in both statistically significant and nonsignificant ways [13]. In a Cochrane Review comparing LMWH and heparinoids to unfractionated heparin, the authors concluded that treatment with LMWH appears to decrease the occurrence of DVT [23, 24]. In an updated version of this Cochrane Review published in 2011 [25], incorporating 9 new trials, comparing either a heparinoid or an LMWH to standard unfractionated heparin, there continues to be a reduction in the odds of developing a DVT in patients treated with either a heparinoid or with LMWH. However, the number of major clinical events such as PE, death, or intracranial hemorrhage was too small to provide estimates as to the risks or benefits of heparinoids or LMWHs compared to standard subcutaneous unfractionated heparin.

It has been shown in surgical patients that the use of graduated compression stockings (GCS) reduces the risk of asymptomatic DVT [26]; this plus other factors, including the risk of intracranial hemorrhage in stroke patients on anticoagulants prompted evaluations of nonpharmacological VTE prophylaxis studies in stoke patients. Besides GCS, other physical methods for VTE prophylaxis include the use of intermittent pneumatic compression. The overall number of clinical studies evaluating nonpharmacological VTE prophylaxis is quite limited compared to the literature of studies using pharmacological means [27].

In summary, patients with an acute ischemic stroke may be at high risk for the development of DVT and subsequent PE, depending on several clinical factors. The development of PE in the hospital setting is 1 of the most important contributors to hospital mortality after a stroke. The use of anticoagulant therapies, such as standard unfractionated heparin, heparinoids, or LMWH can significantly decrease the odds of DVT. There is also a decrease in the odds of PE, although the absolute benefit was small. The use of GCS was not associated with any significant reduction in odds of DVT or death. The use of intermittent pneumatic compression was associated with a nonsignificant trend toward a lower risk of DVT. While awaiting further high-grade data, the use of standardized protocols for DVT prophylaxis using unfractionated heparin or $\mathrm{LMWH}$, reserving mechanical devices for those with a contraindication to heparin, remains an accepted and reasonable therapy.

\section{Hyperglycemia in the Stroke Patient}

There is a high incidence of hyperglycemia in patients with acute neurologic injuries, including traumatic brain injury, subarachnoid hemorrhage, intracerebral hemorrhage, and acute ischemic stroke [28]. More than 30 years ago, in the study by Melamed [29], he described the relative frequency of hyperglycemia after stroke [29]. A recent systematic review examining the incidence of hyperglycemia after stroke found that 8 to $63 \%$ of nondiabetic and 39 to $83 \%$ of diabetic patients with an acute ischemic stroke had hyperglycemia at the time of hospital admission [30].

Hyperglycemia is a common occurrence in patients admitted to the hospital, regardless of when the stroke began and the timing of the glucose measurement [30]. Blood glucoses are initially elevated and then begin to decline in the first day after the acute stroke [31], but the blood glucoses will rise again generally after the first 24 to $88 \mathrm{~h}$, irrespective of whether the patient had known diabetes [32]. There are numerous potential reasons for the occurrence of poststroke hyperglycemia. Patients may have unknown or undiagnosed insulin resistance and impaired glucose control. In a recent study, more than $23 \%$ of acute stroke patients with hospital admission hyperglycemia (who were not previously known to be diabetic) were found to have impaired glucose tolerance and $16 \%$ were newly diagnosed as having diabetes [33]. In addition, acute stroke, as with any other serious acute illness or injury, is a known stressor and therefore apt to activate the hypothalamic-pituitary-adrenal axis [34]. Activation of the hypothalamic-pituitary-adrenal axis will produce a hyperglycemic state from the release of glucocorticoids and activation of the sympathetic nervous system [35].

Melamed [29] was the first to describe the relationship between hyperglycemia and poor clinical outcome after acute ischemic stroke. This relation is now known to be independent of other predictors of poor clinical outcome, such as stroke severity score, infarct size, age, or having diabetes [36]. In the Glycemia in Acute Stroke (GLIAS) trial [37], it was found that an elevated glucose of more 
than $155 \mathrm{mg} / \mathrm{dL}$ in the first 48 hours after an acute ischemic stroke was associated with a 2.7 -fold increase in poor functional outcome, and there was a 3 -fold increase in the risk of death at 3 months [37]. A recent meta-analysis of studies examining hyperglycemia in acute ischemic stroke also reported an increase in mortality rate in patients with hyperglycemia at the time of hospital admission [30]. This finding of poor outcomes with hospital admission hyperglycemia was even more strongly correlated between patients who were treated with the thrombolytic tissue plasminogen activator compared to those who were not treated [38].

There exist no current evidence-based guidelines for glucose control using insulin therapy in patients with an acute ischemic stroke. However, there are several ongoing and planned trials examining the effect of tight glycemic control [35]. In a recent retrospective study, it was demonstrated that early control of hyperglycemia in patients with an acute ischemic stroke could decrease the known deleterious effects of hyperglycemia [39]. In this retrospective study, patients who had received glycemic control therapy with insulin had a 4.6-fold decrease in mortality compared with those patients who had persistent hyperglycemia. This study among others lead to the only current prospective trial thus far completed, the Glucose Insulin in Stroke Trial-United Kingdom (GIST-UK) [40]. This large clinical trial prospectively studied the influence of glycemic control on clinical outcome after an acute stroke [40]. Unfortunately, no definitive conclusions could be drawn from this trial because of several logistical issues. First, the trial was prematurely terminated because of slow patient enrollment, which meant that there was insufficient power to answer the primary study questions. Second, insulin was only infused for a total of only 24 hours. This was much too short to fully assess its effect on glucose control. Third, the trial enrolled stroke patients with both ischemic and hemorrhagic strokes, which likely diluted any potential treatment effect. What was found was that mortality at 90 days was not decreased in the active treatment group and there was a trend toward higher mortality in the insulintreated patients. Outcome measures were found to be similar between the 2 groups, as were the complication rates. Another more recent clinical trial using tight glycemic control only enrolled a small number of patients and therefore was underpowered to assess clinical efficacy [41]. However, in a subset of patients with more severe strokes and much higher admission glucose levels, tight glycemic control was associated with a favorable outcome. In another pilot trial, the Treatment of Hyperglycemia in Ischemic Stroke (THIS) [42] study randomized patients with an acute ischemic stroke to receive continuous insulin infusion to control glucose levels to $<130 \mathrm{mg} / \mathrm{dL}$. This was compared to subcutaneous insulin given every 6 hours for a total of 72 hours. This study found a nonsignificant trend toward improved functional outcomes in the insulin infusion arm of the trial [42].

In summary, hyperglycemia in the acute stroke patient remains a challenge for monitoring, deciding treatment, and capturing outcome data. We now know that hyperglycemia is independently associated with eventual neurologic outcomes, but we don't know if tight glycemic control can alter the eventual outcomes of these patients. Certainly more rigorous, large randomized, controlled trials are necessary to ascertain if the stroke patient will benefit from glycemic control. Currently, the most recent guidelines from the American Heart Association (AHA) [62] offer guidance in regulating blood glucoses for levels $>200 \mathrm{mg}$ / $\mathrm{dL}$, but not to the method of treatment. Education and awareness need to be emphasized and highlighted in the stroke unit.

\section{Treatment for Cerebral Edema and Mass Effect}

\section{Medical Therapies}

\section{Hyperosmolar Therapy}

Hyperosmolar therapy is used to dehydrate the brain and is a key method of treating cerebral edema and mass effect resulting from ischemic stroke. Under normal conditions, aerobic metabolism uses oxygen to produce ATP. However, ischemia will shift glucose metabolism from aerobic and anaerobic. The physiologic result of anaerobic glucose metabolism is a sharp decline in ATP production, which in turn leads to a progressive decrease in many energy dependent cellular functions. One important energy dependent process is the maintenance of appropriate intracellular and extracellular ion concentrations via ATP-dependent ion channels. In particular, loss of ATP will decrease the activity of energy-driven ion channels that regulate the intracellular concentrations of sodium and calcium.

The result of rising intracellular sodium concentration causes the movement of water from the extracellular to intracellular compartment. Along with water movement into the cell, many intracellular pathways are activated as a result of rising intracellular calcium concentration. One important pathway is apoptosis, which will lead to destruction of the cellular and mitochondrial lipid bilayer membrane and eventual cell death. Moreover, from a macroscopic point of view, the result of abnormal intracellular calcium and sodium levels is cellular swelling termed cytotoxic edema. Cytotoxic along with vasogenic and interstitial edema are the 3 most common types of edema patterns recognized in the brain. As cytotoxic edema increases and involves more brain tissue, a growing mass 
effect will produce an area of surrounding penumbra that has threatened viability. This region of tissue is fragile because increasing intracranial mass effect can collapse the microvascular circulation, decrease cerebral perfusion pressure, and decrease cerebral blood flow leading to more tissue ischemia and more edema. All of these processes can act in concert leading to serious lifethreatening brain herniation.

A key to management of cytotoxic edema in stroke is the use of hyperosmolar agents, such as mannitol and hypertonic saline solutions. The goal of hyperosmolar therapy is to mitigate ongoing mass effect and its sequelae by drawing water back into the intravascular space. In addition, animal and human studies suggest additional hemodynamic, neurochemical, and immunologic benefits to hyperosmolar therapy. Hyperosmolar therapy is applied alone or in tandem with established therapies for cerebral edema and intracranial hypertension, which includes elevating the head of the bed, hyperventilation, sedation, fluid resuscitation, and CSF diversion. The following information is aimed to improve the understanding of hyperosmolar therapy use, mechanism of action, and side effects in the acute setting.

Basic Pathophysiology Under normal circumstances, serum osmolarity is well controlled and maintained between 270 and $290 \mathrm{mOsm} / \mathrm{L}$. Aerobic cellular function maintains appropriate extracellular and intracellular oncotic pressures via energy-dependent ion channels, whereas water is free to move passively between compartments. Under normal conditions, the net result of these systems is a steady state that does not result in cellular swelling. With neuronal and cellular ischemia, a new osmotic equilibrium is created, and administration of hyperosmolar solutions aims to be equal, if not exceed, this new oncotic equilibrium; thereby drawing water back into the intravascular space where it cannot contribute to cerebral edema. Hyperosmolar solutions are exogenously given intravenous solutes that aim to dehydrate the swollen brain by setting an osmotic gradient for the movement of water back into the intravascular space. The effectiveness of a given solute to create an osmotic gradient across an intact membrane is described using the osmotic reflection coefficient. Osmotic reflection coefficient is a ratio of the observed to the calculated osmotic pressure generated by a particular solute across a membrane barrier. The range of values given for solutes with a reflection coefficient is 0 to 1 , and those that approach 1 are theoretically optimum. Under normal conditions, sodium has a reflection coefficient of 1 and mannitol is 0.9 . Both are very effective at decreasing brain water content; however, their respective osmotic reflection coefficients are different. As a result, in theory, 1 agent may be superior; however, human studies have not demonstrated this and much controversy remains in the literature as to which agent to choose [43-45]. What is not yet fully understood is how ischemia and the subsequent changes to the blood brain barrier change the calculated osmotic reflection coefficients in vivo.

Pros and Cons of Hyperosmolar Therapy The therapeutic benefits of osmotic agents are not solely limited to brain parenchyma. Hyperosmolar agents influence the movement of water into the intravascular space, leading to hemodilution and expansion of the total vascular volume. Expansion of the intravascular compartment leads to an increase in preload resulting in improved cardiac output and a higher mean arterial pressure. A higher mean arterial pressure may help maintain cerebral microcirculation and provide a neuroprotective effect by limiting ischemic changes to the penumbra. Moreover, increased cerebral blood volume is believed to trigger autoregulatory cerebral vasoconstriction, which, in theory, would decrease total cerebral blood volume and eventually lower intracranial pressure.

Once ischemic changes begin to compromise the blood brain barrier integrity, agents used to counteract cerebral swelling can leak out of the intravascular space and begin to occupy the extracellular space. These exogenous solutes then contribute to the already higher extracellular oncotic pressure created by ischemia and cell death. These combined forces keep brain water content high and further impede water moving back into the intravascular space.

Another important clinical finding of osmotic agent usage is the potential for exacerbation of midline shift. Areas of noninfarcted brain can lose more water content when compared to regions of infarction due to the absence of an intact blood brain barrier. The theory is an intact blood brain barrier allows osmotic agents to realize their full potential and draw a greater amount of water out of the brain when compared to the ischemic hemisphere. This has been shown in a clinical study that used neuroimaging pre-administration and postadministration of mannitol to patients with MCA occlusion [46]. The theoretical risk of potentiating a further shift of infarcted brain toward the noninfarcted hemisphere increases the risk for herniation.

One key consequence of patients exposed to prolonged osmotic agents use is brain adaptation, which is the generation of "organic osmoles" [44, 47, 48] in response to decreasing brain water content. These organic osmoles are postulated to act as a protective adaptation by the brain when faced with serum hypertonicity. The organic osmoles generated remain intracellular, thereby drawing water back from extracellular and intracellular spaces. Clinically this adaptation translates into reduced long-term efficacy of intravenous osmotic agents to maintain their desired effect. Once the decision is made to correct an iatrogenically induced hyperosmolar state, 
caution must be used not to correct serum sodium too quickly with hypotonic solutions. Due to heightened levels of intracellular osmoles, any intravascular water is rapidly drawn into the brain and can lead to rebound cerebral edema and osmotic demyelination.

Hyperosmolar Agent: Mannitol One of the most frequently used osmotic agents is mannitol, which is a very large sugar alcohol that acts as an osmotic agent. Mannitol is excreted unchanged by the kidneys, has a half-life of approximately $2.5 \mathrm{~h}$ [49], and under normal conditions is not able to cross an intact blood brain barrier. Mannitol is typically dosed between 0.5 to $1.5 \mathrm{gm} / \mathrm{kg}$ of body weight in patients with normal renal function. A known side effect to its use is clinically relevant osmotic diuresis. The level of diuresis from serial mannitol administration can lead to intravascular hypovolemia, possibly expanding the area of initial stroke. Therefore, patient volume status should be closely monitored and urine output replaced with intravenous isotonic fluids.

Most of the data used to guide osmotic therapy comes from animal stroke models. One theory is by drawing water back into the cerebral microcirculation, osmotic agents might limit the area of infarction and therefore improve outcome. Further studies have suggested an additional role in reducing infarct size when used with hypothermia [50] and improved postischemic blood flow in animals pretreated with mannitol [51]. One of the first human studies using mannitol came in 1962 when Drs Wise and Chater [52] demonstrated mannitol as a safe and effective agent to decrease intracranial pressure. In 2003, Bereczki et al. [53] performed a prospective observational study of 805 patients reviewing case fatality when mannitol was used in patients admitted within 72 hours of stroke onset. Using a logistic regression model, mannitol did not demonstrate clinical benefit or harm at 1 year. The study was a prospective observational study and not a randomized controlled trial; however, this study did raise concerns about the use of mannitol in acute stroke and did not confirm what was seen in animal models. Then in 2007, Bereczki et al. [45] led a Cochrane Review of mannitol use in acute stroke. This extensive search sought randomized control trials that compared mannitol to a placebo or an open control in patients with acute ischemic stroke or nontraumatic intracerebral hemorrhage. They concluded not enough evidence is available to support the routine use of mannitol in acute stroke patients.

Overall, since 1962 there are surprisingly few randomized control trials that have investigated the use of mannitol in acute stroke. Still, in the face of no substantive data or better alternatives, mannitol is recognized by both the AHA [54] and European Stroke Initiative [55] guidelines as 1 option to treat cerebral edema in acute stroke.
Hypertonic Saline Solution As early as 1919, scientists Weed and McKibben [56] were able to observe the brains of cats shrink when injected with hypertonic saline solution. It was not until the 1980s, in the setting of trauma, when interest toward hypertonic saline solutions gained momentum. In 1993, Vassar et al. [57] evaluated the pre-hospital resuscitation of hypotensive trauma patients using $250 \mathrm{ml}$ of $7.5 \%$ sodium chloride solution, with or without dextran, and compared the hemodynamic parameters to those treated only with Lactated Ringer's Solution. They found improved systolic blood pressure on arrival to the emergency room. In 1997, Wade et al. [58] performed a cohort analysis of patient data from previous prospective, randomized doubleblinded trials that evaluated the use of hypertonic saline/ dextran (HSD) solution in patients who had a traumatic brain injury (TBI). They found patients with TBI who had received the hypertonic saline/dextran solution were twice as likely to survive as those who received standard care. These studies, along with many other animal and human studies, were the basis for further specific research into the cerebrovascular benefits of hypertonic saline solutions.

Currently, hypertonic saline solutions range from 2 to $23.4 \%$ and may vary in administration as a continuous infusion or bolus. The most common hypertonic saline solution used is $3 \% \mathrm{NaCl}$ as a continuous infusion. If faced with rising intracranial pressure, $3 \%$ normal saline can be given through a peripheral intravenously as a $200 \mathrm{ml}$ bolus. This bolus will roughly be equivalent to a bolus of $23.4 \%$ normal saline via central line. The goal when using hypertonic saline is to maintain a serum sodium level of 145 to $155 \mathrm{mEq} / \mathrm{L}$. The most common practice is to hold hypertonic saline and mannitol if the serum sodium reaches $155 \mathrm{mEq} / \mathrm{L}$ or a serum osmolarity of $320 \mathrm{mOsm} / \mathrm{L}$. The serum osmolarity ceiling for hyperosmolar therapy of $320 \mathrm{mOsm} / \mathrm{L}$ has been suggested and is not evidence-based [43].

The immense amount of animal data using hypertonic saline solutions to treat intracranial pathology (TBI with or without hypotension, cerebral edema, elevated ICP, intracranial hemorrhage) seem to share the same proposed clinical benefits with mannitol. Increased cerebral blood volume and oxygen delivery will trigger reflex autoregulatory vasoconstriction of cerebral arteries. This reduction in cerebral blood volume would result in reduced ICP. Further benefits include improved blood flow due to decreasing serum viscosity and improved red blood cell rheology.

Insight into the systemic effects of hypertonic saline is equally important to understanding its cerebrovascular benefits. Hypertonic saline will result in an expansion of the intravascular compartment. Expansion of the intravascular compartment will increase central venous pressure, mean arterial pressure, and cardiac output. Compared to isotonic fluids, resuscitation and expansion of the intravascular 
compartment with hypertonic saline does not require a large volume of fluid to yield the proportionally large increase in mean arterial pressure [59].

In addition, the increase in cardiac output will increase renal perfusion pressure and the glomerular filtration rate. The increase in glomerular filtration rate is seen with both mannitol and hypertonic saline, both leading to diuresis; however, the diuretic effect of mannitol is much more pronounced compared to hypertonic saline, and it can lead to significant hypovolemia. As hypertonic saline increases the intravascular volume, a subsequent humoral influence on the brain and heart results in atrial natriuretic peptide (ANP) production. The exact role of ANP is not well understood, but the net result of both ANP production and presenting the distal tubule with excess sodium load is natriuesis with a mild diuresis. When compared to the diuresis seen with mannitol, hypertonic saline results in much less hypovolemia. This change in the milieu within the nephron also results in a hyperchloremic metabolic acidosis and in hypokalemia.

With respect to immune function, animal studies demonstrate that hypertonic saline can also reduce the inflammatory response and can provide an immunomudulatory effect in TBI models. Activation of the inflammatory cascade occurs after TBI and may lead to migration of cerebral leukocytes to areas of injury. Animal studies investigating the inflammatory response to hemorrhagic shock by Angle et al. [60, 61] found hypertonic saline decreased peripheral leukocyte demargination and adherence. In addition, these studies demonstrated a decrease in hemorrhage-induced neutrophil oxidative burst with hypertonic saline when compared to Lactated Ringer's Solution for resuscitation.

The treatment of patients with cerebral edema due to ischemic stroke uses many approaches. Accepted standard of care includes elevating the head of the bed, short-term hyperventilation, and sedation; whereas some therapies, such as hyperosmolar therapy, are accepted, guidelines suggesting which agent and how much remain sparse. Current data used for interpretation and clinical practice are mostly based on animal models, and the few human studies have not shown clear superiority of 1 agent in comparison to the other. Both mannitol and hypertonic saline improve cerebral hemodynamics, decrease cerebral edema, and may augment cerebral immunology. The differences mostly reside in the route of administration and possible systemic sequelae. Mannitol can be given by peripheral intravenous in an emergency for cerebral herniation syndrome, whereas 23.4\% hypertonic saline requires a central line. However, hypertonic saline can be given in a patient with renal insufficiency and is not a potent diuretic like mannitol.

Moving beyond the specifics of each agent, current published guidelines do not yield any further clues as to which is better or how each should be used. The 2007 AHA [62] acute stroke management guidelines suggest raised intracranial pressure occurs late, if at all, and does not mention hypertonic saline for treatment of cerebral edema. Mannitol is suggested as the only osmotic agent to lower intracranial pressure. In contrast, the 2003 European [55] acute stroke guidelines state cerebral edema can occur within 24 to $48 \mathrm{~h}$ after stroke and support the use of either mannitol or hypertonic saline to treat the early or late rise in intracranial pressure after stroke. The only Cochrane Review assessing evidence-based medicine only exists for mannitol [45] in acute stroke and does not support its routine use. Despite the paucity of randomized data and outcomes, osmotic therapy is common practice in treating those patients with cerebral edema from massive strokes as previously outlined. An important indication is as a bridge or stop-gap therapy to more definitive therapy such as surgical decompression. Further human research is needed to establish which agent is preferred, the dosing regimen for each patient, and efficacy.

\section{Surgical Therapies}

Some patients with proximal MCA ischemic stroke can develop "malignant" swelling and edema, despite medical therapies aimed at lowering intracranial pressure, including sedation, hyperosmolar solutions, hypothermia, and neuromuscular blockade. In 1996, Hanna et al. [63] published a retrospective study that combined clinical presentation, computed tomograpic scan, and mechanism of ischemia to create an "edema risk profile." Patients with 2 of 3 clinical factors (gaze preference, hemiplegia, or hemi-neglect) and evidence of acute cortical infarction on initial computed tomographic scan were highly correlated with the later development of worsening consciousness from edema. In the 1996 study by Hacke et al. [64], patients who had "malignant" MCA infarct develop were found to deteriorate between days 2 and 5. Furthermore, most patients in this study died as a result of transtentorial herniation ipsilateral to the region of ischemia. With a better understanding of the natural history associated with malignant MCA infarct, it became possible to predict which patients may need treatment beyond the best medical management. One definitive method of treatment evolving supratentorial mass effect and rising intracranial pressure is surgical decompression. Surgical decompression involves removing a large portion of the skull, usually extending from the lateral aspect of the frontal bone to the parietal bone ipsilateral to the stroke. Once the bone is removed, a duratomy is performed, which allows for outward brain herniation and decompression of subcortical structures. Figure 1 is an example of a young man who presented with a left MCA 
Fig. 1 The patient is a 28-yearold man who presented with left MCA ischemic stroke. (a, b) Noncontrast axial computed tomographic scans $24 \mathrm{~h}$ from the onset of symptoms. (c, d) Scans $96 \mathrm{~h}$ after admission; note the radiographic signs of septal shift and uncal herniation. (e, f) Scans $24 \mathrm{~h}$ after left hemicraniectomy
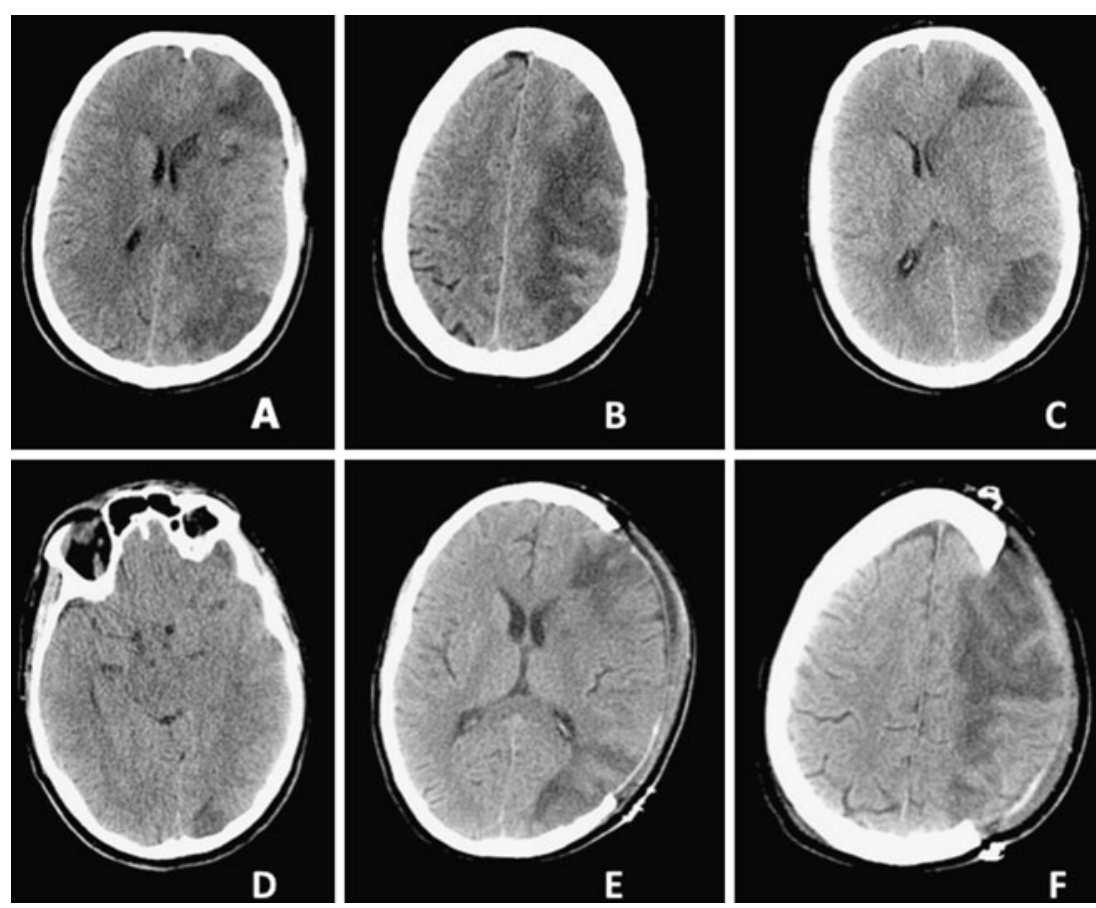

stroke and required a hemicraniectomy to prevent uncal herniation from an evolving malignant cerebral edema.

Long-term safety and the role for hemicraniectomy was studied by the recent French DECIMAL [65], German DESTINY [66] and Dutch HAMLET [67] trials; and collectively evaluated by a pooled analysis [68]. The DECIMAL study [65] enrolled 38 patients with malignant middle cerebral artery infarction from 7 stroke centers and randomized them to receive standard medical therapy or hemicraniectomy plus standard medical therapy. The authors found that at the patients' 1-year follow-up, 15 of 20 had randomized to surgery with an $\mathrm{mRS} \leq 4$ as opposed to only 4 patients in the nonsurgical arm [65]. DESTINY was a concurrent but separate study that focused on mortality at 30 days as the clinical endpoint. Of the 32 patients randomized, 15 of 17 who had received a hemicraniectomy met their primary endpoint $v s 7$ of 15 in the nonsurgical group. Although their study sample was small, they also found a better outcome $(\mathrm{mRS} \leq 3)$ in $47 \%$ of the surgically treated patients $v s 27 \%$ in the nonsurgical group [66]. Vahedi et al. [68] analyzed the randomized patients from the DECIMAL (38 patients) and DESTINY (32 patients) trials in combination with 23 patients from the HAMLET study (which was not complete at the time of this publication), and published a pooled analysis of 93 patients (ages 18-60 with MCA infarct treated within $48 \mathrm{~h}$ ). The primary outcome measure of the pooled data was mRS score at 1 year. Patients who received decompressive surgery compared to control groups had better outcomes ( $75 \%$ vs $24 \%$ with $\mathrm{mRS} \leq 4$ and $43 \%$ vs $23 \%$ with $\mathrm{mRS} \leq 3$ ) [54]. After these publications, the HAMLET investigators presented their final results in 2009. Their goal was to assess the benefit of hemicraniectomy in patients randomized within 4 days rather than $48 \mathrm{~h}$, as with the prior studies. There were 64 patients who were randomized, and in this study surgical decompression was not superior to best medical treatment with $\mathrm{mRS}$ at 1 year. The authors suggested a longer time to randomization and increase in stroke severity (based on median National Institutes of Health Stroke Scale of 24 in the HAMLET vs 21.5 in the DECIMAL study, and 22 in the DESTINY study of the medically treated patients), which may have explained their findings [67].

Although the total number of patients in these studies is small, there seems to be a trend toward better neurological outcome with hemicraniectomy. Beyond the long-term benchmarks based on the modified Rankin scale, the benefits yielded by hemicraniectomy are tangible during a patient's first days in the ICU. The first and most important benefit of hemicraniectomy is improved mortality by preventing transtentorial herniation. Other potential benefits include continued patency of the vascular microcirculation of tissue surrounding the infarction, which may help the surrounding ischemic penumbra from becoming infarcted. Also, mass effect and elevated intracranial pressure without hemicraniectomy can lead to compression of surrounding structures, such as anterior and posterior cerebral arteries leading to additional vascular territories at the risk of infarction, as well as impairment of venous drainage. Finally, hemicraniectomy allows for scaling back of intensive medical therapy needed prior to surgery for the treatment of intracranial hypertension and cerebral edema. 
Suboccipital decompression is the standard treatment for hydrocephalus and brainstem compression [54] caused by either cerebellar infarction or hemorrhage. Unlike MCA infarcts, cerebellar infarction can cause a mass effect that is immediately life threatening. Prior to surgery, the key to management of cerebellar infarcts is remaining 1 step ahead of evolving mass effect. The first radiographic evidence of mass effect created by cerebellar swelling is compression and effacement of the fourth ventricle. As swelling evolves, this process then displaces both the cerebellar tonsils inferiorly and compresses the brainstem against the clivus. Based on a study of 90 patients with cerebellar infarcts, Koh et al. [69] suggests the radiographic order of changes within the posterior fossa is: 1) fourth ventricular compression, 2) fourth ventricular shift, 3) obstructive hydrocephalus, 4) brainstem deformity, and finally 5) basal cistern compression. Based on their study, brainstem compression and hydrocephalus, seen separate or together, were the key radiographic findings that correlate with neurologic decline, and these are the key findings when considering the timing of suboccipital decompression. Prior to suboccipital decompression, placement of an extraventricular drain could help with management of developing hydrocephalus. During the management of these patients, intracranial pressure measurement with an extraventricular drain may not be reliable because a low value may misrepresent the actual amount of local infratentorial pressure actively being generated. Care should be taken not to allow for CSF over drainage from the extraventricular drain as this could create a pressure gradient that could promote rostral herniation of the cerebellarvermis.

Aided by neuroimaging, hemicraniectomy and suboccipital decompression are recognized by both the AHA [62] and European [55] stroke guidelines as definitive therapies to treat brain swelling caused by ischemic stroke. In addition, suboccipital decompression is recognized as the standard of care for the treatment of hydrocephalus and brainstem compression from cerebellar infarction. Hemicraniectomy should be offered early to patients with a malignant MCA infarct to prevent further permanent neurologic complications or death. The key to both hemicraniectomy and suboccipital decompression is appreciating the respective underlying pathologies and having a proactive management strategy. If a patient is $<60$ years of age and the stroke involves the nondominant hemisphere, hemicraniectomy within the first $48 \mathrm{~h}$ of ischemia should be considered; however, the role of hemicraniectomy in patients who are $>60$ years of age present more than $48 \mathrm{~h}$ out or have involvement of the dominant hemisphere is less clear. In these settings, offering hemicraniectomy is a lifesaving measure and the long-term benefit is not fully understood. The risk/benefit profile for patients with a dominant hemisphere stroke should be created on an individual basis, because some will do better than expected [70], including the patient given in Figure 1. Ultimately, the decision to proceed with surgery should account for all available medical information and should also include discussions with family members of the patient when the benefit is less clear.

Acknowledgment Full conflict of interest disclosure is available in the electronic supplementary material for this article.

\section{References}

1. Alberts MJ, Hademenos G, Latchaw RE, et al. for The Brain Attack Coalition. Recommendations for the establishment of primary stroke centers. JAMA 2000;282:3102-3109.

2. Garraway WM. Stroke rehabilitation units: concepts, evaluation, and unresolved issues. Stroke1985;16:178-181.

3. Stroke Unit Trialists' Collaboration. How do stroke units improve patient outcomes? A collaborative systematic review of the randomized trials. Stroke1997a;28:2139-2144.

4. Seenan P, Long M, Langhorn P. Stroke units in their natural habitat. Systematic review of observational studies. Stroke 2007;38:1886-1892.

5. Stroke Unit Trialists' Collaboration. Collaborative systematic review of the randomized trials of organized inpatient (stroke unit) care after stroke. BMJ 1997;314:1151-1159.

6. Stroke Unit Trialists' Collaboration. Organized inpatient (stroke unit) care for stroke. (Cochrane Review). In: The Cochrane Library, 2007:CD000197.

7. Diringer MN, Edwards DF, Mattson DT, et al. Predictors of acute hospital costs for treatment of ischemic stroke in an academic center. Stroke 1999;30:724-728.

8. Ang YH, Chan DKY, Heng DMK, Shen Q. Patient outcomes and length of stay in a stroke unit offering both acute and rehabilitation services. MJA 2003;178:333-336.

9. Taylor WJ, Wong A, Siegert RJ, McNaughton HK. Effectiveness of a clinical pathway for acute stroke care in a district general hospital: an audit. BMC Health Serv Res 2006;6:16.

10. Kwan J, Sandercock P. In-hospital care pathways for stroke: A Cochrane Systematic Review. Stroke 2003:587-588.

11. Rudd AG, Matchar DB. Health policy and outcome research in stroke. Stroke 2004:35:397-400.

12. Ronning OM, Guldvog B, Stavem K. The benefit of an acute stroke unit in patients with intracerebralhaemorrhage: a controlled trial. J Neurol Neurosurg Psychiatry 2001;70:631-634.

13. Kamphuisen PW, Agnelli G, Sebastianelli M. Prevention of venous thromboembolism after acute ischemic stroke. J Thromb Haemost 2005:3:1187-1194.

14. Prins MH, Gelsema R, Sing AK, van Heerde LR, den Ottolander GJ. Prophylaxis of deep venous thrombosis with a low-molecular-weight heparin (Kabi 2165/Fragmin) in stroke patients. Haemostasis 1989;19:245-250.

15. Pambianco, G, Orchard, T, Landau, P. Deep venous thrombosis: prevention in stroke patients during rehabilitation. Arch Phy Med Rehabil 1995;76:324-330.

16. Kelly J, Rudd A, Lewis RR, Coshall C, Moody A, Hunt BJ. Venous thromboembolism after acute ischemic stroke: a prospective study using magnetic resonance direct thrombus imaging. Stroke 2004;35:2320-2325.

17. Geerts WH, Pineo GF, Heit JA, et al. Prevention of venous thromboembolism: the Seventh ACCP Conference on antithrombotic and thrombolytic therapy. Chest 2004;126:338S-400S. 
18. CAST (Chinese Acute Stroke Trial) Collaborative Group. CAST: randomized placebo controlled trial of early aspirin use in 20,000 patients with acute ischaemic stroke. Lancet 1997;349:1641-1649.

19. International Stroke Trial Collaborative Group. The international Stroke Trial (IST): a randomized trial of aspirin, subcutaneous heparin, both, or neither among 19435 patients with acute ischaemic stroke. Lancet 1997;349:1569-1581.

20. Sandercock PAG, Gubitz G, Foley P, Counsell C. Antiplatelet therapy for acute ischaemic stroke (Cochrane Review). The Cochrane Library Issue 4. Article CD000029.

21. McCarthy ST, Turner J. Low dose subcutaneous heparin in the prevention of deep vein thrombosis and pulmonary emboli following acute stroke. Age Ageing 1986;15:84-88.

22. The Trial of ORG 10172 in Acute Stroke Treatment (TOAST) Investigators. Low molecular weight heparinoid ORG 10172 (danaparoid), and outcome after acute ischemic stroke. JAMA 1998;279:1265-1272.

23. Sandercock PAG, Counsell C, Stobbs SL. Low molecular weight heparins or heparinoids versus standard unfractionated heparin for acute ischaemic stroke. Cochrane Database of Systematic Reviews 2005, Issue 2. Art. No. CD000119.

24. Sandercock PAG, Counsell C, Tseng MC. Low molecular weight heparins or heparinoids versus standard unfractionated heparin for acute ischaemic stroke. Cochrane Database of Systematic Reviews 2008, Issue 3. Art. No. CD000119.

25. Sandercock PAG, Counsell C, Tseng MC. Low molecular weight heparins or heparinoids versus standard unfractionated heparin for acute ischaemic stroke. Cochrane Database of Systematic Reviews 2011, Issue 4. Art. No. CD000119.

26. Amaragiri SV, Lees TA. Elastic compression stockings for the prevention of deep vein thrombosis (Cochrane Review). The Cochrane Library Issue 4. Article CD0001484.

27. Naccarato M, Grandi FC, Dennis M, Sandercock PAG. Physical methods for preventing deep vein thrombosis in stroke. Cochrane Database of Systematic Reviews 2010, Issue 8 Article No. CD0001922.

28. Godoy DA, DiNapoli M, Rabinstein AA. Treating hyperglycemia in neurocritical patients: benefits and perils. Neurocrit Care 2010;13;425-438.

29. Melamed E, Reactive hyperglycemia in patients with acute stroke. J NeurolSci 1976;29:267-275.

30. Capes SE, Hunt D, Malberg K, Pathak P, Gerstein HC. Stress hyperglycemia and prognosis of stroke in nondiabetic and diabetic patients: a systematic overview. Stroke 2001:32:2426-2432.

31. Yong M, Kaste M. Dynamic of hyperglycemia as a predictor of stroke outcome in the ECASS-II Trial. Stroke 2008;39:27492755.

32. Allport L, et al. Frequency and temporal profile of poststroke hyperglycemia using continuous glucose monitoring. Diabetes Care 2006;29:1839-44.

33. Matz K, Keresztes K, Tatschl C, et al. Disorders of glucose metabolism in acute stroke patients: an underrecognized problem. Diabetes Care 2006;29:792-797

34. Gauna C, van der Berghe GH, van der Lely AJ. Pituitary function during severe and life threatening illnesses. Pituitary 2005;8:213217.

35. Kruyt ND, Biessels GJ, DeVries JH, Roos YB. Hyperglycemia in acute ischemic stroke: pathophysiology and clinical management. Nat Rev Neurol 2010;6:145-155.

36. Baird TA, et al. Persistent hyperglycemia is independently associated with infarct expansion and worse clinical outcome. Stroke 2003;34:2208-2214.

37. Fuentes B, Castillo J, San Jose B, et al. The prognostic value of capillary glucose levels in acute stroke. Stroke 2009;40;562-568.

38. Bruno A, et al. Admission glucose level and clinical outcomes in the NINDS rt-PA Stroke Trial. Neurology 2002;59;669-674.
39. Gentile NT, Seftchick MW, Huynh T, Kruus LK, Gaughan J. Decreased mortality by normalizing blood glucose after acute ischemic stroke. Acad Emerg Med 2006;13:174-180.

40. Gray CS, et al. Glucose-potassium-insulin infusions in the management of post-stroke hyperglycemia: the UK Glucose Insulin in Stroke Trial (GIST-UK). Lancet 2007;6:397-406.

41. Johnston KC, Hall CE, Kissela BM, Bleck TP, Conaway MR. Glucose regulation in acute stroke patients (GRASP) Trial. A randomized pilot trial. Stroke 2009;40:3804-3809.

42. Bruno A, Kent TA, Coull BM, et al. Treatment of hyperglycemia in ischemic stroke (THIS): a randomized pilot trial. Stroke 2008;39:384-389.

43. Diringer MN, Zazulia AR. Osmotic therapy: fact and fiction. Neurocrit Care 2004;1:219-233.

44. Gullans SR, Verbalis JG. Control of brain volume during hyperosmolar and hypoosmolar conditions. Annu Rev Med 1993;44:289-301.

45. Bereczki D, et al. Mannitol for acute stroke. Cochrane Database Syst Rev 2007;xx:CD001153.

46. Videen TO, et al. Mannitol bolus preferentially shrinks noninfarcted brain in patients with ischemic stroke. Neurology 2001;57:2120-2122.

47. Adrogue HJ, Madias NE. Hypernatremia. N Engl J Med 2000;342:1493-1499.

48. Lien YH, Shapiro JI, Chan L. Effects of hypernatremia on organic brain osmoles. J Clin Invest 1990;85:1427-1435.

49. Rudehill A, et al. Pharmacokinetics and effects of mannitol on hemodynamics, blood and cerebrospinal fluid electrolytes, and osmolality during intracranial surgery. J Neurosurg Anesthesiol 1993;5:4-12.

50. Karibe H, Zarow GJ, Weinstein PR. Use of mild intra-ischemic hypothermia versus mannitol to reduce infarct size after temporary middle cerebral artery occlusion in rats. J Neurosurg 1995;83:93-98.

51. Shirane R, Weinstein PR. Effect of mannitol on local cerebral blood flow after temporary complete cerebral ischemia in rats. $\mathrm{J}$ Neurosurg 1992;76:486-492.

52. Wise BL, Chater N. The value of hypertonic mannitol solution in decreasing brain mass and lowering cerebro-spinal-fluid pressure. J Neurosurg 1962;19:1038-1043.

53. Bereczki D, et al. Mannitol use in acute stroke: case fatality at 30 days and 1 year. Stroke 2003;34:1730-1735.

54. Adams HP Jr, et al. Guidelines for the early management of adults with ischemic stroke: a guideline from the American Heart Association/American Stroke Association Stroke Council, Clinical Cardiology Council, Cardiovascular Radiology and Intervention Council, and the Atherosclerotic Peripheral Vascular Disease and Quality of Care Outcomes in Research Interdisciplinary Working Groups: the American Academy of Neurology affirms the value of this guideline as an educational tool for neurologists. Stroke 2007;38:1655-1711.

55. Olsen TS, et al. European Stroke Initiative Recommendations for Stroke Management-update 2003. Cerebrovasc Dis 2003;16:311-337.

56. Weed LH, McKibben PS. Experimental alteration of brain bulk. Am J Physiol 1919;48:531-535.

57. Vassar MJ, et al. A multicenter trial for resuscitation of injured patients with $7.5 \%$ sodium chloride. The effect of added dextran 70. The Multicenter Group for the Study of Hypertonic Saline in Trauma Patients. Arch Surg 1993;128:1003-1013.

58. Wade CE, et al. Individual patient cohort analysis of the efficacy of hypertonic saline/dextran in patients with traumatic brain injury and hypotension. J Trauma 1997;42(5 suppl):S61-S65.

59. Pascual JM, et al. Resuscitation of intraoperative hypovolemia: a comparison of normal saline and hyperosmotic/hyperoncotic solutions in swine. Crit Care Med 1992;20:200-210.

60. Angle N, et al. Hypertonic saline resuscitation reduces neutrophil margination by suppressing neutrophil L selectin expression. J Trauma 1998;45:7-13. 
61. Angle N, et al. Hypertonic saline resuscitation diminishes lung injury by suppressing neutrophil activation after hemorrhagic shock. Shock 1998;9:164-170.

62. Adams HP Jr, et al. Guidelines for the early management of adults with ischemic stroke: a guideline from the American Heart Association/American Stroke Association Stroke Council, Clinical Cardiology Council, Cardiovascular Radiology and Intervention Council, and the Atherosclerotic Peripheral Vascular Disease and Quality of Care Outcomes in Research Interdisciplinary Working Groups: The American Academy of Neurology affirms the value of this guideline as an educational tool for neurologists. Circulation 2007;115:e478-e534.

63. Hanna JP, et al. Prediction of worsening consciousness from edema after hemispheric infarction. J Stroke Cerebrovasc Dis 1996;6:25-29.

64. Hacke W, et al. "Malignant" middle cerebral artery territory infarction: clinical course and prognostic signs. Arch Neurol 1996;53:309-315.
65. Vahedi K, et al. Sequential-design, multicenter, randomized, controlled trial of early decompressive craniectomy in malignant middle cerebral artery infarction (DECIMAL Trial). Stroke 2007;38:2506-2517.

66. Juttler E, et al. Decompressive Surgery for the Treatment of Malignant Infarction of the Middle Cerebral Artery (DESTINY): a randomized, controlled trial. Stroke 2007;38:2518-2525.

67. Hofmeijer J, et al. Surgical decompression for space-occupying cerebral infarction (the Hemicraniectomy After Middle Cerebral Artery infarction with Life-threatening Edema Trial [HAMLET]): a multicentre, open, randomised trial. Lancet Neurol 2009;8:326-333.

68. Vahedi K, et al. Early decompressive surgery in malignant infarction of the middle cerebral artery: a pooled analysis of three randomised controlled trials. Lancet Neurol 2007;6:215-222.

69. Koh MG, et al. Neuroimaging in deteriorating patients with cerebellar infarcts and mass effect. Stroke 2000;31:2062-2067.

70. Gupta R, et al. Hemicraniectomy for massive middle cerebral artery territory infarction: a systematic review. Stroke 2004;35:539-543. 